\title{
Smooth Transmission over Unsychronized VLC Links
}

\author{
Qifan $\mathrm{Pu}$ \\ University of Science and Technology of China \\ puqf@mail.ustc.edu.cn
}

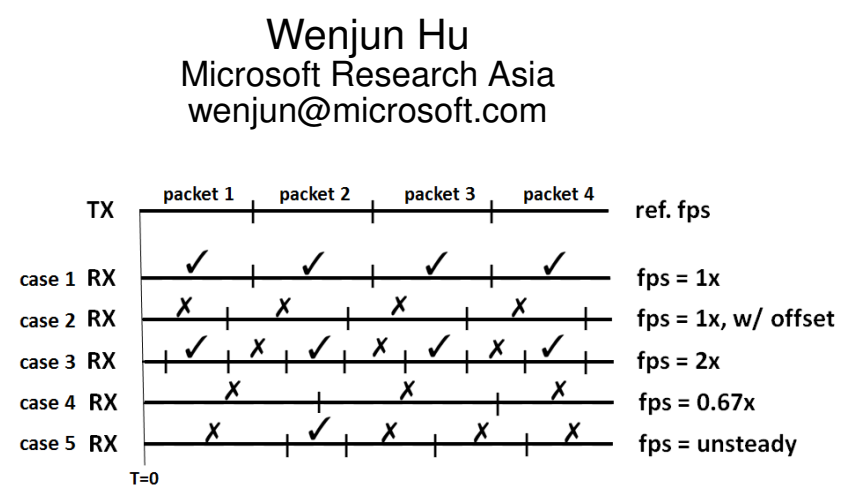

Figure 1: Mismatched FPS between TX-RX pairs. “"” marks the boundary between two consecutive frames. $X$ : "mixed" frames, $\checkmark$ : "clean" frames.

retically, this phone could transmit data at $740 \mathrm{Mbit} / \mathrm{s}$ and receive at over $3 \mathrm{Gbit} / \mathrm{s}$.

However, real systems are hindered by imperfect frame synchronization, image distortion, and computational complexity, which result in much lower observed data rates of, say, 172 bps [4]. While previous research often focused on reducing image distortion, we try to solve the basic synchronization problem in such a system.

Lack of synchronization between a transmitter and receiver happens mainly due to mismatched frame rates and a phase offset between the screen and the camera. Figure 1 shows several examples. Typically, only frames staying within the period of one transmission are clean (marked " $\checkmark$ "), and thus decodable. If a captured spans across different TX frames, it becomes a mixed frame (marked " $X$ ") and is normally dropped. Only after receiving all the transmitted frames "cleanly" can a receiver successfully decode the original message, as in cases 1 and 3.

With a traditional coding scheme, a receiver should capture frames at a rate at least twice that of the transmitter's display frame rate to get all frames (case 3). Unfortunately, the opposite often happens in practice. Modern LCD/AMOLED screens usually have a $60 \mathrm{fps}$ frame rate or at least $30 \mathrm{fps}$, while cameras have lower and more diverse frame rates in the range of 24 to $30 \mathrm{fps}$. Moreover, many smartphone cameras exhibit unstable frame rates because of the hardware design and firmware settings. Our tests on a series of Android phones show that the camera will automatically adjust its frame rate according to the lighting conditions. It is neither desirable nor possible to control the frame rate at the application level. The system performance can be significantly bottlenecked by the low, fluctuating frame rates at the camera end.

To achieve synchronization, previous systems like PixNet 3 and COBRA [4] simply reduce the screen frame rate to ensure that the receiver can pick out a good frame every other frame. Langlotz et al [2] embed 2D barcodes into time-multiplexed 3D barcodes by repeating frames on multiple, orthogonal color channels, essentially limiting the transmitter's frame rate. These approaches are inefficient because the transmitter side capacity is under-utilized and the receiver drops a large number of "mixed" frames.

This paper introduces rateless coding into a VLC system and describes both an algorithm and a frame format design to solve the synchronization problem. The idea is to encode an original message into a series transmitted frames, such that mixed frames 


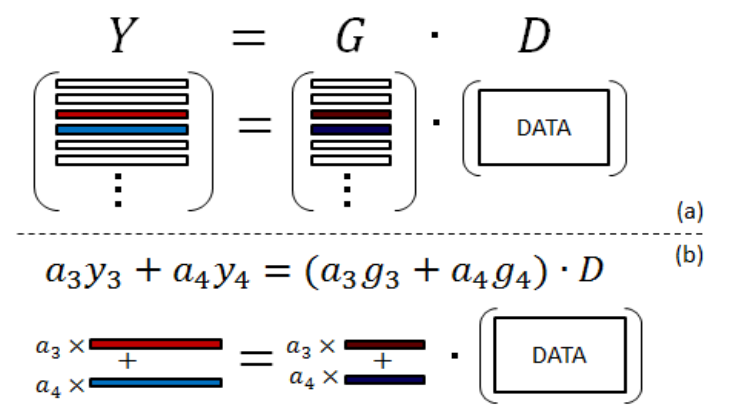

Figure 2: The last step in the whole encoding chain. (a) An encoded frame $Y$ is generated from the original message $D$ with an encoding matrix $G$. (b) Mixed frames are still useful.

can still be used to recover the original message, provided that the frame indices are properly tracked.

\section{SMOOTH CODES}

Smooth Codes adds two steps to the conventional encoding process to generate image frames. First, after the original messages are encoded into a data matrix, we generate frames by calculating projections of the data matrix, similar to the rateless coding approach in Strider [1]. Second, each encoded frame is prefixed with a tracking bar to aid decoding. The receiver calculates the weights of the original frames based on the tracking bars and the encoding matrix, and then decodes the original frames.

\subsection{Coding Algorithm}

The coding process can be represented by a matrix multiplication (Figure 2]a)). The input data message is assembled into a matrix $D$ and multiplied by a pseudo-random encoding matrix $G$ to generate an output matrix $Y$. Each row of $Y$ is a single TX frame and is transmitted in one time slot:

$$
Y=G \cdot D \quad \text { or } \quad \vec{y}_{i}=\vec{g}_{i} \cdot D
$$

$G$ is randomly generated and normalized, so each frame is a distinct projection of the original message. Note that $G$ can have infinitely many rows.

The $i^{t h}$ frame in $Y$ is related to the $i^{\text {th }}$ row of $G$ alone but not to any other row of $G$. The equation will hold true no matter how we reorder the rows of $Y$ and $G$ consistently. This means that the receiver only needs enough frames to decode the original data, irrespective of which frames are received and in what order. The number of frames needed is related with the channel distortion and noise level. The encoding matrix $G$ can be generated offline and is known to the receiver. The actual decoding is straightforward, and mainly requires inverting the encoding matrix.

If the receiver gets a combination of TX frames, $\vec{y}_{\text {mixed }}$, we have:

$$
\vec{y}_{\text {mixed }}=\sum_{i=p}^{q} a_{i} \vec{y}_{i}=\sum_{i=p}^{q} a_{i} \vec{g}_{i} D=\left(\sum_{i=p}^{q} a_{i} \vec{g}_{i}\right) \cdot D=\vec{g}_{\text {mixed }} \cdot D
$$

where $\vec{y}_{i}$ is the $i_{t h}$ frame in the combination and $a_{i}$ is its weight, and $\vec{g}_{\text {mixed }}$ serves as the corresponding random encoding vector for $\vec{y}_{\text {mixed }}$. Most importantly, $\vec{y}_{\text {mixed }}$ is still a distinct projection of $D$ just as any other "clean" RX frame. If the corresponding $\vec{g}_{\text {mixed }}$ can be found, we can use the mixed packets instead of dropping them. We now discuss how to get $\vec{g}_{\text {mixed }}$.

\subsection{Frame Tracking}

Although the above encoding process allows us to recover messages from any subset of the transmitted frames, we still need to know the indices of the frames to identify the appropriate rows of the original $G$ for decoding. Furthermore, in the case of "mixed"

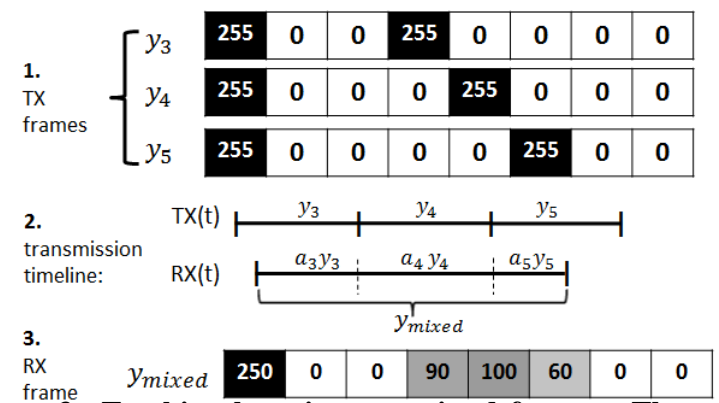

Figure 3: Tracking bars in transmitted frames. The anchor block and a frame-specific tag block are set to 255 . The receiver estimates the weights of original frames based on the received values of those blocks. The weights in this received frame are $a_{3}=\frac{90}{250}, a_{4}=\frac{100}{250}, a_{5}=\frac{60}{250}$.

frames, we also need to track the weight, $a_{i}$, of each transmitted frame, then we can calculate $\vec{g}_{\text {mixed }}$ from $a_{i}$ and $g_{i}$. In our solution, we obtain these indices and weights by adding tracking bars in each transmitted frame. These are analogous to known training sequences and pilots in 802.11 frames.

Figure 3 shows the structure of a tracking bar. The first block of the tracking bar is always tagged by the same color across frames to serve as an anchor. Then each frame will tag one block from the remaining blocks with the same anchor color. The tagged block in$\operatorname{dex} s_{i}=\bmod \left(y_{i}, M A X \_B L O C K S\right)$, where $y_{i}$ is the frame index, and $M A X \_B L O C K S$ is such that a receiver with the lowest capture rate can still distinguish the frames. The receiver can infer the composition of a received frame by estimating the weights of the original frames using the received values of the tracking bar blocks.

\section{PRELIMINARY RESULTS}

We have implemented a prototype as a proof of concept. Our system consists of a laptop screen as the transmitter, a phone camera as a receiver, and an offline decoder. The Lenovo X201i laptop takes the original data, encodes them with a randomly generated encoding matrix $G$, assembles the encoded data into frames, and displays them at an arbitrary frame rate. The camera from an HTC EVO 3D takes pictures of the screen with an arbitrary shutter speed, and feeds all the received images into the offline decoder. We vary the frame rates at both ends to assess the decoding quality under a wide range of channel conditions. Initial results show that Smooth Codes achieves a much higher throughput than previous approaches.

In future work, we will aim to build a real-time phone-to-phone VLC system based on Smooth Codes, including an immediate feedback mechanism. We will also build applications leveraging such VLC links.

\section{REFERENCES}

[1] A. Gudipati and S. Katti. Strider: automatic rate adaptation and collision handling. In Proceedings of the ACM SIGCOMM 2011 conference, 2011.

[2] T. Langlotz and O. Bimber. Unsynchronized 4D Barcodes Coding and Decoding Time-Multiplexed 2D Colorcodes. 2007.

[3] S. D. Perli, N. Ahmed, and D. Katabi. Pixnet: Lcd-camera pairs as communication links. In Proceedings of the ACM SIGCOMM 2010 Conference on Applications, Technologies, Architectures, and Protocols for Computer Communications. ACM, 2010.

[4] G. X. Tian Hao, Ruogu Zhou. Cobra: Color barcode streaming for smartphone systems. In Proceedings of the 10th International Conference on Mobile Systems, Applications, and Services (MobiSys). ACM, 2012. 